\title{
Clustering of color sources and the shear viscosity of the QGP in heavy ion collisions at RHIC and LHC energies
}

\author{
J. Dias de Deus ${ }^{1}$, A.S. Hirsch ${ }^{2}$, C. Pajares ${ }^{3}$, R.P. Scharenberg ${ }^{2}$, B.K. Srivastava ${ }^{2, a}$ \\ ${ }^{1}$ CENTRA, Instituto Superior Tecnico, 1049-001 Lisbon, Portugal \\ ${ }^{2}$ Department of Physics, Purdue University, West Lafayette, IN 47907, USA \\ ${ }^{3}$ Departamento de Fisica de Particulas, Universidale de Santiago de Compostela and Instituto Galego de Fisica de Atlas Enerxias (IGFAE), \\ 15782 Santiago, de Compostela, Spain
}

Received: 24 May 2012 / Revised: 2 July 2012 / Published online: 28 August 2012

(C) The Author(s) 2012. This article is published with open access at Springerlink.com

\begin{abstract}
We present our results on the shear viscosity to entropy ratio $(\eta / s)$ in the framework of the clustering of the color sources of the matter produced at RHIC and LHC energies. The onset of de-confinement transition is identified by the spanning percolating cluster in 2D percolation. The relativistic kinetic theory relation for $\eta / s$ is evaluated using the initial temperature $(T)$ and the mean free path $\left(\lambda_{\mathrm{mfp}}\right)$. The analytic expression for $\eta / s$ covers a wide temperature range. At $T \sim 150 \mathrm{MeV}$ below the hadron to QGP transition temperature of $\sim 168 \mathrm{MeV}$, with increasing temperatures the $\eta / s$ value drop sharply and reaches a broad minimum $\eta / s \sim 0.20$ at $T \sim 175-185 \mathrm{MeV}$. Above this temperature $\eta / s$ grows slowly. The measured values of $\eta / s$ are $0.204 \pm 0.020$ and $0.262 \pm 0.026$ at the initial temperature of $193.6 \pm 3 \mathrm{MeV}$ from central $\mathrm{Au}+\mathrm{Au}$ collisions at $\sqrt{s_{N N}}=200 \mathrm{GeV}$ (RHIC) and 262.2 $\pm 13 \mathrm{MeV}$ in central $\mathrm{Pb}+\mathrm{Pb}$ collisions at $\sqrt{s_{N N}}=2.76 \mathrm{TeV}$ (LHC). These $\eta / s$ values are 2.5 and 3.3 times the AdS/CFT conjectured lower bound $1 / 4 \pi$ but are consistent with theoretical $\eta / s$ estimates for a strongly coupled QGP.
\end{abstract}

\section{Introduction}

The observation of the large elliptic flow at RHIC in noncentral heavy ion collisions suggest that the matter created is a nearly perfect fluid with a very low shear viscosity [1-4]. Recently, attention has been focused on the shear viscosity to entropy density ratio $\eta / s$ as a measure of the fluidity [5-8]. The observed temperature averaged $\eta / s$, based on viscous hydrodynamics analyses of RHIC data, are suggestive of a strongly coupled plasma $[9,10]$. The effect of the bulk viscosity is expected to be negligible. It has been

\footnotetext{
a e-mail: brijesh@purdue.edu
}

conjectured, based on infinitely coupled super-symmetric Yang-Mills (SYM) gauge theory using the correspondence between Anti de-Sitter(AdS) space and conformal field theory (CFT), that the lower bound for $\eta / s$ is $1 / 4 \pi$ and is the universal minimal viscosity to entropy ratio even for QCD [11]. However, there are theories in which this lower bound can be violated [12]. In this work, we use the color string percolation model (CSPM) $[13,14]$ to obtain $\eta / s$ as a function of the temperature above and below the hadron to $\mathrm{QGP}$ transition. The measured $\eta / s$ values are for $\mathrm{Au}+\mathrm{Au}$ collisions at $\sqrt{s_{N N}}=200 \mathrm{GeV}$ at RHIC and for $\mathrm{Pb}+\mathrm{Pb}$ collisions at $\sqrt{s_{N N}}=2.76 \mathrm{TeV}$ at LHC.

\section{Clustering of color sources}

Multiparticle production is currently described in terms of color strings stretched between the projectile and the target, which decay into new strings and subsequently hadronize to produce observed hadrons. Color strings may be viewed as small areas in the transverse plane filled with color field created by colliding partons. With growing energy and size of the colliding system, the number of strings grows, and they start to overlap, forming clusters, in the transverse plane very much similar to disks in two dimensional percolation theory. At a certain critical density a macroscopic cluster appears that marks the percolation phase transition. This is the Color String Percolation Model (CSPM) [13, 14]. The interaction between strings occurs when they overlap and the general result, due to the $\mathrm{SU}(3)$ random summation of charges, is a reduction in multiplicity and an increase in the string tension hence increase in the average transverse momentum squared, $\left\langle p_{t}^{2}\right\rangle$. We assume that a cluster of $n$ strings that occupies an area of $S_{n}$ behaves as a single color source with a higher color field $Q_{n}$ corresponding to the vectorial sum of the color charges of each individual string $\boldsymbol{Q}_{\mathbf{1}}$. 
The resulting color field covers the area of the cluster. As $\boldsymbol{Q}_{\boldsymbol{n}}=\sum_{1}^{n} \boldsymbol{Q}_{1}$, and the individual string colors may be oriented in an arbitrary manner respective to each other, the average $Q_{1 i} Q_{1 j}$ is zero, and $Q_{n}^{2}=n Q_{1}^{2}$.

Knowing the color charge $\boldsymbol{Q}_{n}$ one can obtain the multiplicity $\mu$ and the mean transverse momentum squared $\left\langle p_{t}^{2}\right\rangle$ of the particles produced by a cluster of $n$ strings [14]

$\mu_{n}=\sqrt{\frac{n S_{n}}{S_{1}}} \mu_{0} ; \quad\left\langle p_{t}^{2}\right\rangle=\sqrt{\frac{n S_{1}}{S_{n}}}\left\langle p_{t}^{2}\right\rangle_{1}$

where $\mu_{0}$ and $\left\langle p_{t}^{2}\right\rangle_{1}$ are the mean multiplicity and $\left\langle p_{t}^{2}\right\rangle$ of particles produced from a single string with a transverse area $S_{1}=\pi r_{0}^{2}$. For strings just touching each other $S_{n}=n S_{1}$, and $\mu_{n}=n \mu_{0},\left\langle p_{t}^{2}\right\rangle_{n}=\left\langle p_{t}^{2}\right\rangle_{1}$. When strings fully overlap, $S_{n}=S_{1}$ and therefore $\mu_{n}=\sqrt{n} \mu_{0}$ and $\left\langle p_{t}^{2}\right\rangle_{n}=\sqrt{n}\left\langle p_{t}^{2}\right\rangle_{1}$, so that the multiplicity is maximally suppressed and the $\left\langle p_{t}^{2}\right\rangle_{n}$ is maximally enhanced. This implies a simple relation between the multiplicity and transverse momentum $\mu_{n}\left\langle p_{t}^{2}\right\rangle_{n}=n \mu_{0}\left\langle p_{t}^{2}\right\rangle_{1}$, which means conservation of the total transverse momentum produced.

In the thermodynamic limit, one obtains an analytic expression $[13,14]$

$\left\langle\frac{n S_{1}}{S_{n}}\right\rangle=\frac{\xi}{1-e^{-\xi}} \equiv \frac{1}{F(\xi)^{2}}$

where $F(\xi)$ is the color suppression factor. With $F(\xi) \rightarrow 1$ as $\xi \rightarrow 0$ and $F(\xi) \rightarrow 0$ as $\xi \rightarrow \infty$, where $\xi=\frac{N_{s} S_{1}}{S_{N}}$ is the percolation density parameter. Equation (1) can be written as $\mu_{n}=n F(\xi) \mu_{0}$ and $\left\langle p_{t}^{2}\right\rangle_{n}=\left\langle p_{t}^{2}\right\rangle_{1} / F(\xi)$. The critical cluster which spans $S_{N}$, appears for $\xi_{c} \geq 1.2$ [15]. It is worth noting that CSPM is a saturation model similar to the Color Glass Condensate (CGC), where $\left\langle p_{t}^{2}\right\rangle_{1} / F(\xi)$ plays the same role as the saturation momentum scale $Q_{s}^{2}$ in the CGC model [16-18].

\section{Experimental determination of the color suppression factor $F(\xi)$}

The suppression factor is determined by comparing the $p p$ and $\mathrm{A}+\mathrm{A}$ transverse momentum spectra. To evaluate the initial value of $\xi$ from data for $\mathrm{Au}+\mathrm{Au}$ collisions, a parameterization of $p p$ events at $200 \mathrm{GeV}$ is used to compute the $p_{t}$ distribution [19-21]

$d N_{c} / d p_{t}^{2}=a /\left(p_{0}+p_{t}\right)^{\alpha}$

where a is the normalization factor. $p_{0}$ and $\alpha$ are parameters used to fit the data. This parameterization also can be used for nucleus-nucleus collisions to take into account the interactions of the strings [14]

$d N_{c} / d p_{t}^{2}=\frac{a^{\prime}}{\left(p_{0} \sqrt{F\left(\xi_{p p}\right) / F(\xi)}+p_{t}\right)^{\alpha}}$
The color suppression factor $F(\xi)$ is related to the percolation density parameter $\xi$.

$F(\xi)=\sqrt{\frac{1-e^{-\xi}}{\xi}}$

In pp collisions $F(\xi) \sim 1$ at these energies due to the low overlap probability.

In this way the STAR analysis of charged hadrons obtained the preliminary results for the percolation density parameter, $\xi$ at RHIC for several collisions systems as a function of centrality [19]. Figure 1 shows a plot of $F(\xi)$ as a function of charged particle multiplicity per unit transverse area $\frac{d N_{c}}{d \eta} / S_{N}$ for $\mathrm{Au}+\mathrm{Au}$ collisions at $200 \mathrm{GeV}$ for various centralities for the STAR data [20,21]. The error on $F(\xi)$ is $\sim 3 \%$. $F(\xi)$ decreases in going from peripheral to central collisions. The $\xi$ value is obtained using Eq. (5), which increases with the increase in centrality. The fit to the $\mathrm{Au}+\mathrm{Au}$ points has the functional form

$F(\xi)=\exp \left[-0.165-0.094 \frac{d N_{c}}{d \eta} / S_{N}\right]$

The STAR results for $\mathrm{Au}+\mathrm{Au}$ collisions at $\sqrt{s_{N N}}=$ $200 \mathrm{GeV}$ can be used to estimate $\mathrm{F}(\xi)$ values for $\mathrm{Pb}+\mathrm{Pb}$ collisions at different centralities using the fit function given by Eq. (6) for $\mathrm{Au}+\mathrm{Au}$. Recently, the ALICE experiment at LHC published the charged-particle multiplicity density data as a function of centrality in $\mathrm{Pb}+\mathrm{Pb}$ collisions at $\sqrt{s_{N N}}=2.76 \mathrm{TeV}$ [22]. The ALICE data points are shown in Fig. 1. For central $0-5 \%$ in $\mathrm{Pb}+\mathrm{Pb}$ collisions $\xi=10.56$ as compared to $\xi=2.88$ for central $\mathrm{Au}+\mathrm{Au}$ collisions at $200 \mathrm{GeV}$. For $\mathrm{Au}+\mathrm{Au}$ central collisions we have found that the Bjorken energy density $\varepsilon$ in the collision is proportional to $\xi$. To evaluate $\varepsilon$ the charged pion multiplicity at mid rapidity and the Schwinger QED2 production time

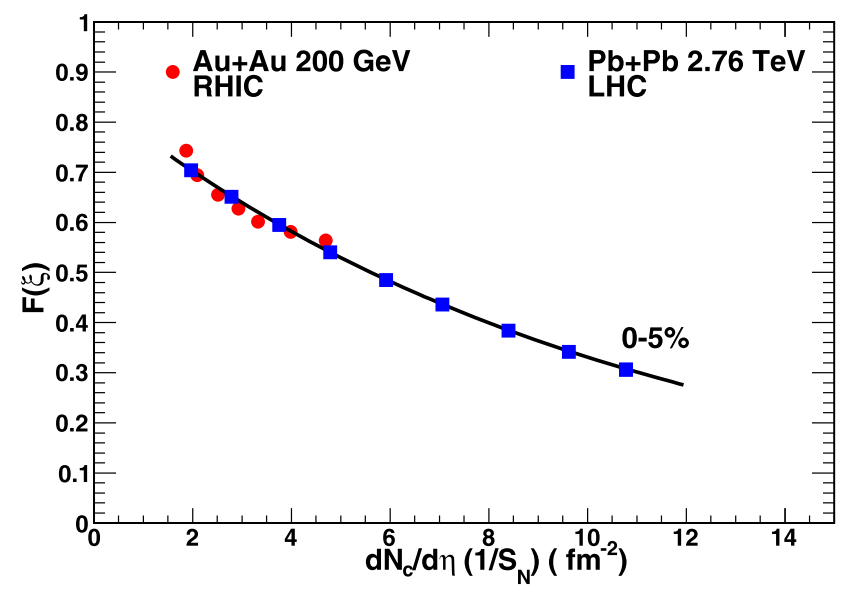

Fig. 1 Color suppression factor $F(\xi)$ as a function of $\frac{d N_{c}}{d \eta} / S_{N}\left(\mathrm{fm}^{-2}\right)$. The solid red circles are for $\mathrm{Au}+\mathrm{Au}$ collisions at $200 \mathrm{GeV}$ (STAR data) [19]. The error is smaller than the size of the symbol. The line is fit to the STAR data. The solid blue squares are for $\mathrm{Pb}+\mathrm{Pb}$ at $2.76 \mathrm{TeV}$ 


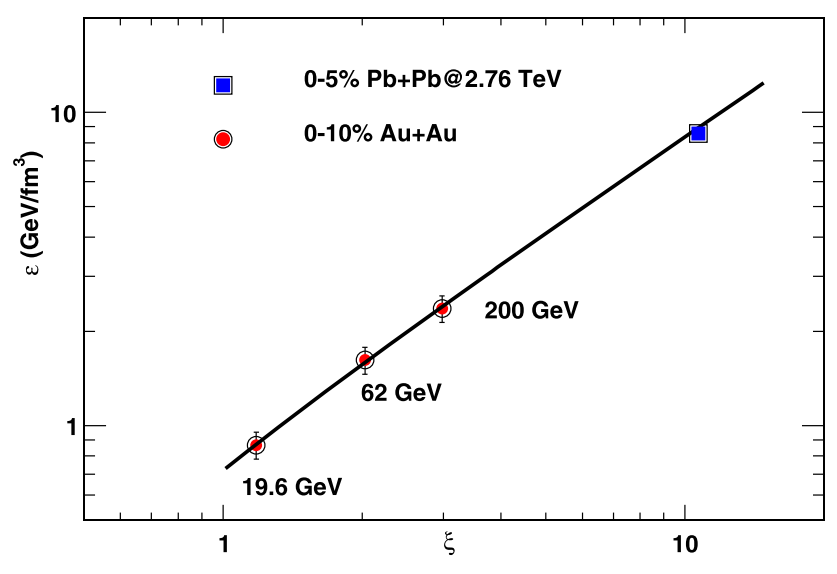

Fig. 2 Energy density $\epsilon$ as a function of the percolation density parameter $\xi$. The value for LHC energy is shown as blue square

were used [21, 23]. Figure 2 shows a plot of energy density as a function of $\xi . \varepsilon=0.788 \xi$ for the range $1.2<\xi<2.88$. The extrapolated value of $\varepsilon$ for central $\mathrm{Pb}+\mathrm{Pb}$ collision at $2.76 \mathrm{TeV}$ is $8.32 \mathrm{GeV} / \mathrm{fm}^{3}$ as shown in Fig. 2.

\section{Determination of the temperature}

The connection between the measured $\xi$ and the temperature $T(\xi)$ involves the Schwinger mechanism (SM) for particle production. The Schwinger distribution for massless particles is expressed in terms of $p_{t}^{2}[24,25]$

$d n / d p_{t}^{2} \sim e^{-\pi p_{t}^{2} / x^{2}}$

where the average value of the string tension is $\left\langle x^{2}\right\rangle$. The tension of the macroscopic cluster fluctuates around its mean value because the chromo-electric field is not constant. The origin of the string fluctuation is related to the stochastic picture of the QCD vacuum. Since the average value of the color field strength must vanish, it can not be constant but changes randomly from point to point [26]. Such fluctuations lead to a Gaussian distribution of the string tension for the cluster, which transforms SM into the thermal distribution [26]

$d n / d p_{t}^{2} \sim e^{\left(-p_{t} \sqrt{\frac{2 \pi}{\left\langle x^{2}\right\rangle}}\right)}$

with $\left\langle x^{2}\right\rangle=\pi\left\langle p_{t}^{2}\right\rangle_{1} / F(\xi)$.

The temperature is expressed as [27]

$T(\xi)=\sqrt{\frac{\left\langle p_{t}^{2}\right\rangle_{1}}{2 F(\xi)}}$

Recently, it has been suggested that fast thermalization in heavy ion collisions can occur through the existence of an event horizon caused by a rapid de-acceleration of the colliding nuclei [28]. The thermalization in this case is due to the Hawking-Unruh effect $[29,30]$. In CSPM the strong color field inside the large cluster produces de-acceleration of the primary $q \bar{q}$ pair which can be seen as a thermal temperature by means of the Hawking-Unruh effect. The string percolation density parameter $\xi$ which characterizes the percolation clusters measures the initial temperature of the system. Since this cluster covers most of the interaction area, this temperature becomes a global temperature determined by the string density. In this way at $\xi_{c}=1.2$ the connectivity percolation transition at $T\left(\xi_{c}\right)$ models the thermal deconfinement transition.

We adopt the point of view that the experimentally determined universal chemical freeze-out temperature $\left(T_{f}\right)$ is a good measure of the phase transition temperature, $T_{c}$ [31]. $\left\langle p_{t}^{2}\right\rangle_{1}$ is evaluated using Eq. (9) at $\xi_{c}=1.2$ with $T_{f}=167.7 \pm 2.6 \mathrm{MeV}$ [32]. This gives $\sqrt{\left\langle p_{t}^{2}\right\rangle_{1}}=207.2 \pm$ $3.3 \mathrm{MeV}$ which is close to $\simeq 200 \mathrm{MeV}$ used in a previous calculation of the percolation transition temperature [27]. This calibrates the CSPM temperature scale. The dynamics of massless particle production has been studied in QED2 quantum electrodynamics. QED2 can be scaled from electrodynamics to quantum chromodynamics using the ratio of the coupling constants. Here the production time for a boson (gluon) is $\tau_{\text {pro }}=\frac{2.405 \hbar}{m c^{2}}$ [25]. This gives $\tau_{\text {pro }} \sim 1.13 \mathrm{fm}$ for central $\mathrm{Au}+\mathrm{Au}$ collisions at $\sqrt{s_{N N}}=200 \mathrm{GeV}$. The temperature obtained using Eq. (9) was $\sim 193.6 \mathrm{MeV}$ for $\mathrm{Au}+\mathrm{Au}$ collisions. For $\mathrm{Pb}+\mathrm{Pb}$ collisions the temperature is $\sim 262.2 \mathrm{MeV}$ for $0-5 \%$ centrality, which is expected to be $\sim 35 \%$ higher than the temperature from $\mathrm{Au}+\mathrm{Au}$ collisions [21]. A recent summary of the results from $\mathrm{Pb}+\mathrm{Pb}$ collisions at the LHC has mentioned that the initial temperature increases at least by $30 \%$ as compared to the top RHIC energy [33]. Table 1 gives the CSPM values $\xi, T, \varepsilon$ and $\eta / s$ at $T / T_{c}=0.88,1,1.16$ and 1.57 .

One way to verify the validity of extrapolation from RHIC to LHC energy is to compare the energy density expressed as $\varepsilon / T^{4}$ with the available lattice QCD results. Figure 3 shows a plot of $\varepsilon / T^{4}$ as a function of $T / T_{c}$. The lat-

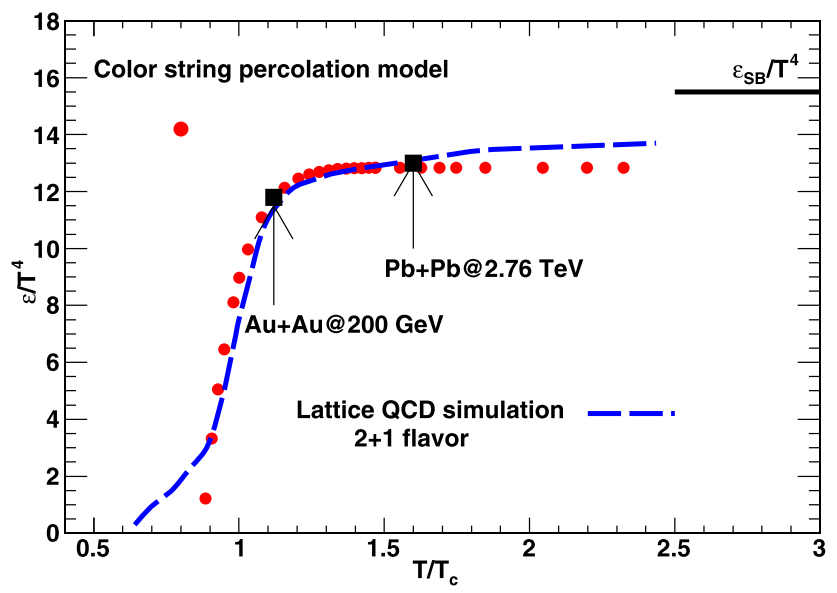

Fig. $3 \varepsilon / T^{4}$ as a function of $T / T_{c}$. The lattice QCD calculation is shown as dotted blue line [34] 
Table 1 The measured percolation density parameter $\xi$, temperature $T$, energy density $\varepsilon$ and $\eta / s$ for the meson gas [36]; the hadron to QGP transition; $\mathrm{Au}+\mathrm{Au}$ at $200 \mathrm{GeV}$ and $\mathrm{Pb}+\mathrm{Pb}$ at $2.76 \mathrm{TeV}$ (estimated). $\mathrm{Au}+\mathrm{Au}$ is for $0-10 \%$ and $\mathrm{Pb}+\mathrm{Pb}$ is for $0-5 \%$ central events

\begin{tabular}{lllll}
\hline System & $\xi$ & $T(\mathrm{MeV})$ & $\varepsilon\left(\mathrm{GeV} / \mathrm{fm}^{3}\right)$ & $\eta / s$ \\
\hline Meson Gas & 0.22 & 150.0 & - & 0.76 \\
Hadron to QGP & 1.2 & $167.7 \pm 2.6$ & $0.94 \pm 0.07$ & $0.240 \pm 0.012$ \\
$\mathrm{Au}+\mathrm{Au}$ & $2.88 \pm 0.09$ & $193.6 \pm 3.0$ & $2.27 \pm 0.16$ & $0.204 \pm 0.020$ \\
$\mathrm{~Pb}+\mathrm{Pb}$ & $10.56 \pm 1.05$ & $262.2 \pm 13.0$ & $8.32 \pm 0.83$ & $0.260 \pm 0.026$ \\
\hline
\end{tabular}

tice QCD results are from HotQCD Collaboration [34]. It is observed that at LHC energy the CSPM results are in excellent agreement with the lattice QCD results. The lattice and CSPM results are available for $T / T_{c}<2$.

\section{Shear viscosity}

The relativistic kinetic theory relation for the shear viscosity over entropy density ratio, $\eta / s$ is given by $[9,35]$

$\frac{\eta}{S} \simeq \frac{T \lambda_{\mathrm{mfp}}}{5}$

where $T$ is the temperature and $\lambda_{\mathrm{mfp}}$ is the mean free path given by

$\lambda_{\mathrm{mfp}} \sim \frac{1}{\left(n \sigma_{t r}\right)}$

$n$ is the number density of an ideal gas of quarks and gluons and $\sigma_{t r}$ the transport cross section for these constituents.

After the cluster is formed it behaves like a free gas of constituents. Equation (10) can be applied to obtain the shear viscosity. In CSPM the number density is given by the effective number of sources per unit volume

$n=\frac{N_{\text {sources }}}{S_{N} L}$

$L$ is the longitudinal extension of the source, $L=1 \mathrm{fm}$ [27].

The area occupied by the strings is related to $\xi$ through the relation $\left(1-e^{-\xi}\right) S_{N}$. Thus the effective no. of sources is given by the total area occupied by the strings divided by the effective area of the string $S_{1} F(\xi)$.

$N_{\text {sources }}=\frac{\left(1-e^{-\xi}\right) S_{N}}{S_{1} F(\xi)}$

In general $N_{\text {sources }}$ is smaller than the number of single strings. $N_{\text {sources }}$ equals the number of strings $N_{s}$ in the limit of $\xi=0$. The number density of sources from Eqs. (12) and (13) becomes

$n=\frac{\left(1-e^{-\xi}\right)}{S_{1} F(\xi) L}$

In CSPM the transport cross section $\sigma_{t r}$ is the transverse area of the effective string $S_{1} F(\xi)$. Thus $\sigma_{t r}$ is directly pro- portional to $F(\xi)$ and hence to $\frac{1}{T^{2}}$. The mean free path is given by

$\lambda_{\mathrm{mfp}}=\frac{L}{\left(1-e^{-\xi}\right)}$

For a large value of $\xi$ the $\lambda_{\text {mfp }}$ reaches a constant value. $\eta / s$ is obtained from $\xi$ and the temperature

$\frac{\eta}{s}=\frac{T L}{5\left(1-e^{-\xi}\right)}$

Well below $\xi_{c}$, as the temperature increases, the string density increases and the area is filled rapidly and $\lambda_{\text {mfp }}$ and $\eta / s$ decrease sharply. Above $\xi_{c}$, more than $2 / 3$ of the area are already covered by strings, and therefore the area is not filling as fast and the relatively small decrease of $\lambda_{\mathrm{mfp}}$ is compensated by the rising of temperature, resulting in a smooth increase of $\eta / s$. The behavior of $\eta / s$ is dominated by the fractional area covered by strings. This is not surprising because $\eta / s$ is the ability to transport momenta at large distances and that has to do with the density of voids in the matter.

\section{Results and discussion}

Figure 4 shows a plot of $\lambda_{\mathrm{mfp}}, T$ and $\lambda_{\mathrm{mfp}} \times T$ as a function of $\xi$. Thus the product $T(\xi) \times \lambda_{\text {mfp }}$ will have a minimum in $\eta / s$. It has been shown that $\eta / s$ has a minimum at the critical point for various substances for example helium, nitrogen and water [10]. Thus the measurement of $\eta / s$ as a function of temperature can indicate the critical point in the QCD phase diagram with $T \sim 175-185 \mathrm{MeV}$.

Figure 5 shows a plot of $\eta / s$ as a function of $T / T_{c}$. The estimated value of $\eta / s$ for $\mathrm{Pb}+\mathrm{Pb}$ is also shown in Fig. 5 at $T / T_{c}=1.57$. The lower bound shown in Fig. 5 is given by AdS/CFT [11]. These results from STAR and ALICE data show that the $\eta / s$ value is 2.5 and 3.3 times the KSS bound [11].

The theoretical estimates of $\eta / s$ has been obtained as a function of $T / T_{c}$ for both the weakly (wQGP) and strongly (sQGP) coupled QCD plasma are shown in Fig. 5 [9]. It is seen that at the RHIC top energy $\eta / s$ is close to the sQGP. Even at the LHC energy it follows the trend of the sQGP. By extrapolating the $\eta / s$ CSPM values to higher temperatures it is clear that $\eta / s$ could approach the weak coupling limit near $T / T_{c} \sim 5.8$. The CSPM $\eta / s$ value for the 


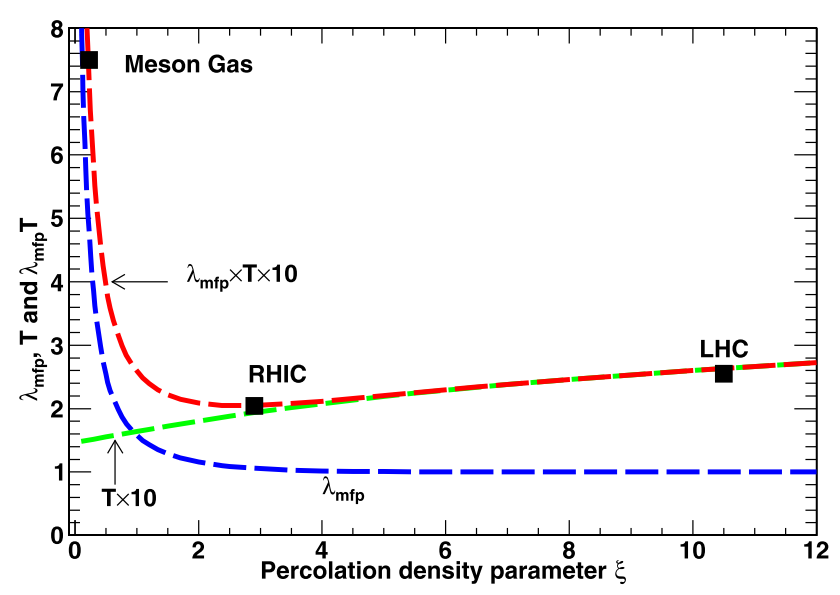

Fig. $4 \lambda_{\mathrm{mfp}}$ in fm (blue line), temperature in $\mathrm{GeV}$ scaled by a factor of 10 (green line) and $\lambda_{\mathrm{mfp}} \times$ scaled $T$ in $\mathrm{GeV}$ fm (red line) as a function of $\xi$. The minimum in $\eta / s$ is due to the combination of $\lambda_{\mathrm{mfp}}$ and $T$. Meson gas [36], RHIC and LHC points are also shown as solid black square

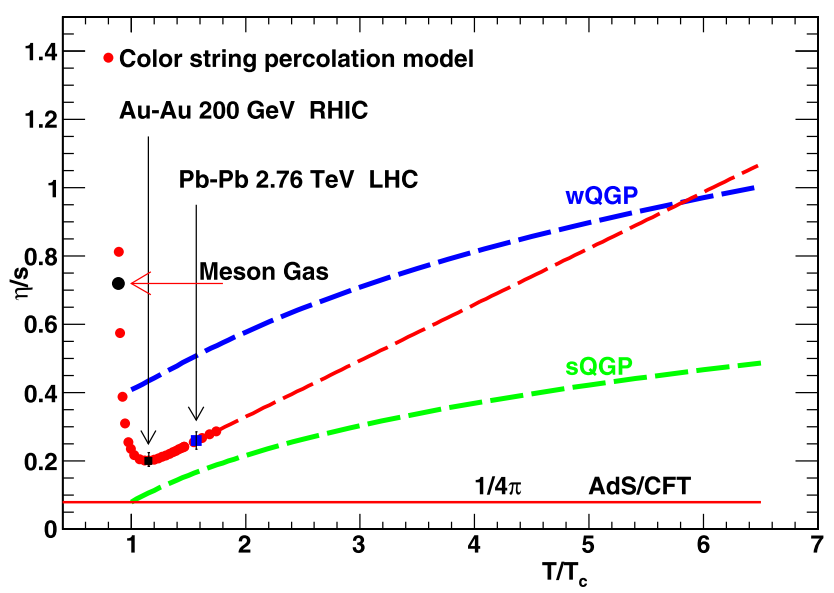

Fig. $5 \eta / s$ as a function of $T / T_{c}$. $\mathrm{Au}+\mathrm{Au}$ at $200 \mathrm{GeV}$ for $0-10 \%$ centrality is shown as solid black square. WQGP and sQGP values are shown as dotted blue and green lines respectively [9]. The estimated value for $\mathrm{Pb}+\mathrm{Pb}$ at $2.76 \mathrm{TeV}$ for $0-5 \%$ centrality is shown as a solid blue square. The red dotted line represents the extrapolation to higher temperatures from the CSPM. The hadron gas value for $\eta / s \sim 0.7$ is shown as solid black circle at $T / T_{c} \sim 0.88$ [36]

hadron gas is in agreement with the calculated value using measured elastic cross sections for a gas of pions and kaons [37]. $\eta / s$ has also been obtained in several other calculations for pure glue matter [38], in the semi quark qluon plasma [39] and in quasiparticle description [40]. In pure SU(3) gluodynamics a conservative upper bound for $\eta / s$ was obtained $\eta / s=0.134(33)$ at $T=1.65 T_{c}$ [41]. In the quasiparticle approach also low $\eta / s \sim 0.2$ is obtained for $T>1.05 T_{c}$ and rises very slowly with the increase in temperature [42]. In CSPM also $\eta / s$ grows with temperature as $0.16 T / T_{c}$.
The CSPM model calculations have also successfully described the elliptic flow and the nuclear modification factor at RHIC and LHC energies [43]. In addition CSPM has determined the equation of state of the QGP and the bulk thermodynamic value of $\varepsilon / T^{4}$ and $s / T^{3}$ in excellent agreement with Lattice Gauge calculations [21]. This emphasizes the quantitative nature of the CSPM when applied to the data at $\sim 1 \mathrm{TeV}$ scale.

\section{Summary}

In summary the relativistic kinetic theory relation for shear viscosity to entropy density ratio $\eta / s=\frac{1}{5} T \lambda_{\text {mfp }}$ was evaluated as a function of the temperature using the measured transverse momentum spectra and the Color String Percolation Model. The color suppression factor $F(\xi)$ was extracted from the transverse momentum spectrum of charged hadrons. We found $\eta / s=0.204 \pm 0.020$ at $T / T_{c}=1.15$ (RHIC) and $\eta / s=0.260 \pm 0.020$ at $T / T_{c}=1.57$ (LHC). In the phase transition region $\eta / s$ is $2-3$ times the conjectured quantum limit for RHIC to LHC energies. The whole picture is consistent with the formation of a fluid with a low shear to viscosity ratio. The percolation framework provides us with a microscopic picture which predicts the early thermalization required for hydrodynamical calculations.

The minimum in $\eta / s$ can be studied as a function of the beam energy at RHIC that could locate the critical point/crossover in the QCD phase diagram seen in substances like helium, nitrogen and water $[6,10]$. The accurate determination of $\eta / s$ is also important for the evaluation of another transport coefficient, the jet quenching parameter $\hat{q}$ $[44,45]$.

Acknowledgements This research was supported by the Office of Nuclear Physics within the U.S. Department of Energy Office of Science under Grant No. DE-FG02-88ER40412.

J.D.D. thanks the support of the FCT/Portugal project PPCDT/ FIS/575682004. C.P. was supported by the project FPA2011-022776 of MICINN the Spanish Consolider Ingenio 2010 program CPAN and Conselleria Education Xunta de Galicia.

Open Access This article is distributed under the terms of the Creative Commons Attribution License which permits any use, distribution, and reproduction in any medium, provided the original author(s) and the source are credited.

\section{References}

1. I. Arsene et al. (BRAHAMS Collaboration), Nucl. Phys. A 757, 1 (2005)

2. B.B. Back et al. (PHOBOS Collaboration), Nucl. Phys. A 757, 28 (2005)

3. J. Adams et al. (STAR Collaboration), Nucl. Phys. A 757, 102 (2005) 
4. K. Adcox et al. (PHENIX Collaboration), Nucl. Phys. A 757, 184 (2005)

5. D. Teaney, Phys. Rev. C 68, 034913 (2003)

6. T. Schafer, D. Teaney, Rep. Prog. Phys. 72, 126001 (2009)

7. R.A. Lacey et al., Phys. Rev. Lett. 98, 092301 (2007)

8. P. Romatschke, U. Romatschke, Phys. Rev. Lett. 99, 272301 (2007)

9. T. Hirano, M. Gyulassy, Nucl. Phys. A 769, 71 (2006)

10. L.P. Csernai, J.I. Kapusta, L.D. McLerran, Phys. Rev. Lett. 97, 152303 (2006)

11. P.K. Kovtun, D.T. Son, A.O. Starinets, Phys. Rev. Lett. 94, 111601 (2005)

12. A. Buchel, R.C. Myers, A. Sinha, J. High Energy Phys. 03, 084 (2009)

13. M.A. Braun, C. Pajares, Eur. Phys. J. C 16, 349 (2000)

14. M.A. Braun, F. del Moral, C. Pajares, Phys. Rev. C 65, 024907 (2002)

15. H. Satz, Rep. Prog. Phys. 63, 1511 (2000)

16. L. McLerran, R. Venugopalan, Phys. Rev. D 49, 2233 (1994)

17. L. McLerran, R. Venugopalan, Phys. Rev. D 49, 3352 (1994)

18. J. Dias de Deus, C. Pajares, Phys. Lett. B 695, 455 (2011)

19. B.K. Srivastava, R.P. Scharenberg, T. Tarnowsky (STAR Collaboration), Nukleonika 51, s109 (2006)

20. B.I. Abelev et al. (STAR Collaboration), Phys. Rev. C 79, 34909 (2009)

21. R.P. Scharenberg, B.K. Srivastava, A.S. Hirsch, Eur. Phys. J. C 71, $1510(2011)$

22. K. Aamodt et al. (ALICE Collaboration), Phys. Rev. Lett. 106, 032301 (2011)

23. J.D. Bjorken, Phys. Rev. D 27, 140 (1983)

24. J. Schwinger, Phys. Rev. 128, 2425 (1962)
25. C.Y. Wong, Introduction to High Energy Heavy Ion Collisions (1994), p. 289

26. A. Bialas, Phys. Lett. B 466, 301 (1999)

27. J. Dias de Deus, C. Pajares, Phys. Lett. B 642, 455 (2006)

28. D. Kharzeev, E. Levin, K. Tuchin, Phys. Rev. C 75, 044903 (2007)

29. S.W. Hawking, Commun. Math. Phys. 43, 199 (1975)

30. W.G. Unruh, Phys. Rev. D 14, 870 (1976)

31. P. Braun-Munzinger, J. Stachel, C. Wetterich, Phys. Lett. B 596, 61 (2004)

32. F. Becattini, P. Castorina, A. Milov, H. Satz, Eur. Phys. J. C 66, 377 (2010)

33. B. Muller, J. Schukraft, W. Wyslouch, arXiv:1202.3233 [hep-exp]

34. A. Bazavov et al., Phys. Rev. D 80, 014504 (2009)

35. P. Danielewicz, M. Gyulassy, Phys. Rev. D 31, 53 (1985)

36. D. Teaney, in $Q G P 4$, ed. by R.C. Hwa, X.N. Wang (World Scientific, Singapore, 2010), p. 207

37. M. Prakash et al., Phys. Rep. 227, 321 (1993)

38. A.S. Khvorostukhin, V.D. Toneev, D.N. Voskresensky, Phys. Rev. C 83, 035204 (2011)

39. Y. Hidaka, R.D. Pisarski, Phys. Rev. D 81, 076002 (2010)

40. M. Bluhm, B. Kampfer, K. Redlich, arXiv:1011.5634 [hep-ph]

41. H.B. Meyer, Phys. Rev. D 76, 101701(R) (2007)

42. A. Peshier, W. Cassing, Phys. Rev. Lett. 94, 172301 (2005)

43. I. Bautista, J. Dias de Deus, C. Pajares, arXiv:1102.3837 [hep-ph]

44. A. Majumder, B. Muller, X.-N. Wang, Phys. Rev. Lett. 99, 192301 (2007)

45. J. Casalderrey-Solana, X.-N. Wang, Phys. Rev. C 77, 024902 (2008) 